\title{
Searches for the Anomalous FCNC Top-Higgs Couplings with Polarized Electron Beam at the LHeC
}

\author{
XiaoJuan Wang, Hao Sun, and Xuan Luo \\ Institute of Theoretical Physics, School of Physics \& Optoelectronic Technology, Dalian University of Technology, \\ No. 2 Linggong Road, Dalian, Liaoning 116024, China \\ Correspondence should be addressed to Hao Sun; haosun@dlut.edu.cn
}

Received 29 September 2016; Revised 2 December 2016; Accepted 4 January 2017; Published 16 March 2017

Academic Editor: Burak Bilki

Copyright (c) 2017 XiaoJuan Wang et al. This is an open access article distributed under the Creative Commons Attribution License, which permits unrestricted use, distribution, and reproduction in any medium, provided the original work is properly cited. The publication of this article was funded by SCOAP ${ }^{3}$.

\begin{abstract}
We study the single top and Higgs associated production $e^{-} p \rightarrow v_{e} \bar{t} \rightarrow v_{e} h \bar{q}(h \rightarrow b \bar{b})$ in the top-Higgs FCNC couplings at the $\mathrm{LHeC}$ with the electron beam energy of $E_{e}=60 \mathrm{GeV}$ and $E_{e}=120 \mathrm{GeV}$ and combination of a $7 \mathrm{TeV}$ and $50 \mathrm{TeV}$ proton beam. With the possibility of $e$-beam polarization $\left(p_{e}=0, \pm 0.6\right)$, we distinct the cut-based method and the multivariate analysis- (MVA-) based method and compare with the current experimental and theoretical limits. It is shown that the branching ratio $\mathrm{Br}(t \rightarrow u h)$ can be probed to $0.113(0.093 \%), 0.071(0.057 \%), 0.030(0.022 \%)$, and $0.024(0.019 \%)$ with the cut-based (MVA-based) analysis at $\left(E_{p}, E_{e}\right)$ $=(7 \mathrm{TeV}, 60 \mathrm{GeV}),\left(E_{p}, E_{e}\right)=(7 \mathrm{TeV}, 120 \mathrm{GeV}),\left(E_{p}, E_{e}\right)=(50 \mathrm{TeV}, 60 \mathrm{GeV})$, and $\left(E_{p}, E_{e}\right)=(50 \mathrm{TeV}, 120 \mathrm{GeV})$ beam energy and $1 \sigma$ level. With the possibility of $e$-beam polarization, the expected limits can be probed down to $0.090(0.073 \%), 0.056(0.045 \%), 0.024$ $(0.018 \%)$, and $0.019(0.015 \%)$, respectively.
\end{abstract}

\section{Introduction}

The Large Hadron Electron Collider (LHeC) is the second electron-hadron collider following HERA [1]. With remarkable higher energy and luminosity, the $\mathrm{LHeC}$ is a major step towards understanding the Higgs physics and QCD. For the $\mathrm{LHeC}$ colliding energy, the $7 \mathrm{TeV}$ proton beam at the LHC as well as the $50 \mathrm{TeV}$ proton beam at the future FCC-he [2] and a new $60 \mathrm{GeV}$ electron beam [1] are envisaged. To probe new physics, the anomalous flavor changing neutral current (FCNC) Yukawa interactions, between the top-Higgs and either an up or charm quark, would provide a clear signal. The SM Lagrangian can be extended by the following terms:

$$
\mathscr{L}=\kappa_{t u h} \bar{t} u h+\kappa_{t c h} \bar{t} c h+\text { h.c., }
$$

where the real parameters $\kappa_{t u h}$ and $\kappa_{t c h}$ denote the FCNC couplings of the Higgs to up-type quarks. The total decay width of the top quark $\Gamma_{t}$ is

$$
\Gamma_{t}=\Gamma_{t \rightarrow W^{-} b}^{\mathrm{SM}}+\Gamma_{t \rightarrow c h}+\Gamma_{t \rightarrow u h}
$$

where the decay width $\Gamma_{t \rightarrow W^{-} b}^{\mathrm{SM}}$ and $\Gamma_{t \rightarrow u(c) h}$ can be found in $[3,4]$, respectively. Thus, the branching ratio for $t \rightarrow u(c) h$ can be approximately given by

$$
\begin{aligned}
\operatorname{Br}(t \longrightarrow u(c) h) & =\frac{\kappa_{t u(c) h}^{2}}{\sqrt{2} G_{F} m_{t}^{2}} \frac{\left(1-\tau_{h}^{2}\right)^{2}}{\left(1-\tau_{W}^{2}\right)^{2}\left(1+2 \tau_{W}^{2}\right)} \\
& \approx 0.512 \kappa_{t u(c) h}^{2},
\end{aligned}
$$

where $G_{F}$ is the Fermi constant and $\tau_{W}=m_{W} / m_{t}$. The $W$ boson and top quark masses are chosen to be $m_{W}=$ $79.82 \mathrm{GeV}$ and $m_{t}=173.2 \mathrm{GeV}$, respectively.

Up to now, the investigation of $t \rightarrow q h$ anomalous couplings has been experimented by many groups, which gives the strong limits on the top-Higgs FCNC couplings. For instance, according to the ATLAS and CMS collaborations, the upper limits of $\operatorname{Br}(t \rightarrow q h)<0.79 \%[5,6]$ and $\operatorname{Br}(t \rightarrow$ $q h)<0.45 \%$ [7] have been set at $95 \%$ confidence level (CL). At one-loop level, the $D^{0}-\overline{D^{0}}$ mixing observable can receive sizeable contributions with such an unvanishing flavor violating tqH coupling [8]. Using data observed on $D^{0}-\overline{D^{0}}$, 
TABLE 1: The results from different experimental and phenomenological channels.

\begin{tabular}{lcc}
\hline Channels & Data set & Limits \\
\hline$t \bar{t} \rightarrow W b q h \rightarrow \ell \nu b \gamma \gamma q$ & ATLAS, $4.7(20.3) \mathrm{fb}^{-1} @ 7(8) \mathrm{TeV}$ & $\operatorname{Br}(t \rightarrow q h)<0.79 \%[5,6]$ \\
$t \bar{t} \rightarrow W b q h \rightarrow \ell \nu b \gamma \gamma q$ & $\mathrm{Br}(t \rightarrow u h)<0.45 \%[7]$ \\
$D^{0}-\overline{D^{0}}$ mixing data & $\mathrm{CMS}, 19.5 \mathrm{fb}^{-1} @ 8 \mathrm{TeV}$ & $\mathrm{Br}(t \rightarrow q h)<0.5 \%[8]$ \\
$Z \rightarrow c \bar{c}$ and $E W$ observables & - & $\operatorname{Br}(t \rightarrow q h)<0.21 \%[9]$ \\
$W t \rightarrow W h q \rightarrow \ell \nu b \gamma \gamma q$ & - & $\operatorname{Br}(t \rightarrow q h)<0.24 \%[10]$ \\
$t \bar{t} \rightarrow W b q h \rightarrow \ell \nu b \gamma \gamma q$ & $\operatorname{Br}(t \rightarrow u h)<0.23 \%[11]$ \\
$t \bar{t} \rightarrow t q h \rightarrow \ell \nu b b \bar{b} q$ & LHC, $3000 \mathrm{fb}^{-1} @ 14 \mathrm{TeV}, 3 \sigma$ & $\operatorname{Br}(t \rightarrow q h)<0.112 \%[12]$ \\
$t h \rightarrow \ell \nu b \tau^{+} \tau^{-}$ & LHC, $3000 \mathrm{fb}^{-1} @ 14 \mathrm{TeV}$ & $\operatorname{Br}(t \rightarrow u h)<0.15 \%[13]$ \\
$t h \rightarrow \ell \nu b \ell^{+} \ell^{-} X$ & ILC, $3000 \mathrm{fb}^{-1} @ 500 \mathrm{GeV}$ & $\operatorname{Br}(t \rightarrow u h)<0.22 \%[13]$ \\
$t h \rightarrow j j b b \bar{b}$ & LHC, $100 \mathrm{fb}^{-1} @ 13 \mathrm{TeV}$ & $\operatorname{Br}(t \rightarrow u h)<0.36 \%[13]$ \\
\hline
\end{tabular}

the upper limit of $\operatorname{Br}(t \rightarrow q h)<5 \times 10^{-3}$ can be achieved. Furthermore, through $Z \rightarrow c \bar{c}$ decay and electroweak observables, the upper limit of $\operatorname{Br}(t \rightarrow q h)<0.21 \%$ [9] can be obtained.

On the other hand, based on the experimental data, many phenomenological studies are performed from different channels. For instance, [10] found that the branching ratios $\mathrm{Br}(t \rightarrow q h)$ can be probed to $0.24 \%$ at $3 \sigma$ level at $14 \mathrm{TeV}$ LHC with integrated luminosity of $3000 \mathrm{fb}^{-1}$ through the process $W t \rightarrow W h q \rightarrow \ell \nu b \gamma \gamma q$. Reference [11] explored the topHiggs FCNC couplings through $t \bar{t} \rightarrow W b q h \rightarrow \ell v b \gamma \gamma q$ and found that the branching ratios $\operatorname{Br}(t \rightarrow u h)$ can be probed to $0.23 \%$ at $3 \sigma$ sensitivity at $14 \mathrm{TeV}$ LHC with $=3000 \mathrm{fb}^{-1}$. And [12] obtained $\operatorname{Br}(t \rightarrow q h)$ to be $0.112 \%$ based on the process of $t \bar{t} \rightarrow t q h \rightarrow \ell \nu b b \bar{b} q$. The process of $t h \rightarrow \ell \nu b \tau^{+} \tau^{-}$has been studied in [13] and the authors therein estimated the upper limits of $\operatorname{Br}(t \rightarrow u h)<0.15 \%$ at $100 \mathrm{fb}^{-1}$ of $13 \mathrm{TeV}$ data for multilepton searches. The results from different experiments and theoretical channels are summarized in Table 1.

In this study, we examined $e^{-} p \rightarrow \nu_{e} \bar{t} \rightarrow v_{e} h \bar{q}$ at the LHeC, where the Higgs boson decays to $b \bar{b}$ at 7 (50) TeV with 60 (120) $\mathrm{GeV}$ electron beam and $1000 \mathrm{fb}^{-1}$ integrated luminosity. The possibility of $e$-beam polarization is also considered. The Feynman diagram is plotted in Figure 1. The main backgrounds which yield the same or similar final states to the signal are listed as follows:

$$
\begin{aligned}
& e^{-} p \longrightarrow v_{e}\left(\bar{t} \longrightarrow\left(W^{-} \longrightarrow j j\right) \bar{b}\right), \\
& e^{-} p \longrightarrow e^{-} j j j, \\
& e^{-} p \longrightarrow v_{e} j j j, \\
& e^{-} p \longrightarrow v_{e}(h \longrightarrow b \bar{b}) j, \\
& e^{-} p \longrightarrow v_{e}(z \longrightarrow b \bar{b}) j,
\end{aligned}
$$

where $j=g, u, \bar{u}, d, \bar{d}, c, \bar{c}, s, \bar{s}, b$, and $\bar{b}$ if possible. Notice that $e^{-} p \rightarrow e^{-} j j j$ is the neutral current multijet QCD background, and all the others belong to charged current (CC) productions. For the single top background $e^{-} p \rightarrow$ $\nu_{e}\left(\bar{t} \rightarrow\left(W^{-} \rightarrow j j\right) \bar{b}\right)$, the produced top quark will decay to a $W$ boson and a $b$-jet. The $W$ boson continues to decay to non- $b$-jet final states, which might be mistagged as a $b$-jet. With the same final states, $e^{-} p \rightarrow \nu_{e}(h \rightarrow b \bar{b}) j$ and $e^{-} p \rightarrow$ $\nu_{e}(z \rightarrow b \bar{b}) j$ are the irreducible backgrounds corresponding to associated Higgs jet and $Z$ jet which contain three QED couplings. $e^{-} p \rightarrow v_{e} j j j$ is the CC multijet QCD background. Similar to the single top background, misidentification of one or more of the final state light jets to $b$-jet makes this process a reducible background.

\section{Tools and Method}

During the simulation, we first extract the Feynman Rules by using the FeynRules package [14] and generate the event with MadGraph@NLO [15]. PYTHIA6.4 [16] was set to solve the initial and final state parton shower, hadronization, heavy hadron decays, and so forth. We use CTEQ6L $[17,18]$ as the parton distribution function and set the renormalization and factorization scale to be $\mu_{r}=\mu_{f}$. We take the input heavy particle masses as $m_{h}=125.7 \mathrm{GeV}, m_{t}=173.2 \mathrm{GeV}, m_{Z}=$ $91.1876 \mathrm{GeV}$, and $m_{W}=79.82 \mathrm{GeV}$, respectively. We employ the following basic preselections cuts to select the events:

$$
\begin{aligned}
E_{T}^{\text {missing }} & \geq 15 \mathrm{GeV}, \\
p_{T}^{k_{0}} & \geq 15 \mathrm{GeV}, \quad k_{0}=j, b, \ell, \\
\left|\eta^{j}\right| & <5, \\
\left|\eta^{b}\right| & <5, \\
\left|\eta^{\ell}\right| & \leq 3, \\
\Delta R\left(k_{1} k_{2}\right) & >0.4, \quad k_{1} k_{2}=j j, j \ell, j b, b b, b \ell,
\end{aligned}
$$




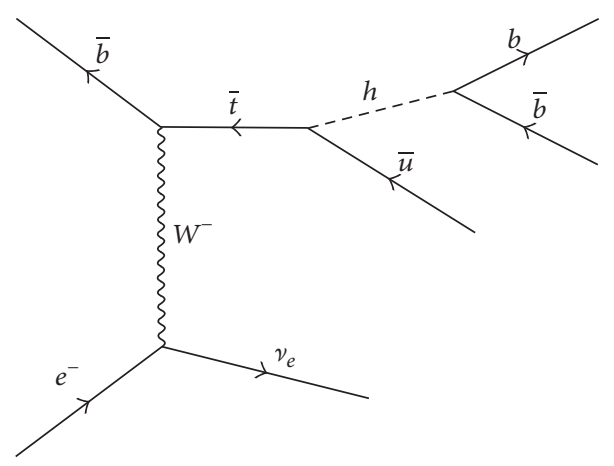

FIGURE 1: Feynman diagram for the partonic process $e^{-} \bar{b} \rightarrow v_{e} \bar{t} \rightarrow v_{e} h \bar{q} \rightarrow v_{e} b \bar{b} \bar{q}$ at the LHeC through flavor changing top-Higgs interactions.

where $\Delta R=\sqrt{\Delta \Phi^{2}+\Delta \eta^{2}}$ is the separation with $\Delta \eta$ and $\Delta \Phi$ in the rapidity-azimuth plane and $p_{T}^{\text {jet, } b, \ell}$ and $\left|\eta^{\text {jet, } b, \ell}\right|$ are the transverse momentum and the pseudorapidity of jets, $b$-jets, and leptons, while $E_{T}^{\text {missing }}$ is the missing transverse momentum. Then we adopt a cut-based method and a multivariate analysis- (MVA-) based method for signal and background analysis, respectively.

2.1. Cut-Based Method. In order to distinguish between signal-related events and background-related events as much as possible, we set a series of cuts. We list all the cut-based selections here:

(i) Cut 1: the basic preselection cuts

(ii) Cut 2: the selection $e^{-} p \rightarrow E_{T}^{\text {missing }}+0 \ell+\geq 3$ jets, (with at least 2 tagged $b$-jets)

(iii) Cut 3: missing transverse energy $E_{T}^{\text {missing }}>20 \mathrm{GeV}$

(iv) Cut 4: the reconstructed top quark mass window $m_{t} \in$ [148 GeV, $178 \mathrm{GeV}$ ]

(v) Cut 5: the reconstructed $W$ boson mass window $m_{W}<50 \mathrm{GeV}$ or $m_{W}>90 \mathrm{GeV}$

(vi) Cut 6: the reconstructed $Z$ boson mass window $m_{Z}<$ $55 \mathrm{GeV}$ or $m_{Z}>95 \mathrm{GeV}$

(vii) Cut 7: the reconstructed Higgs mass window $m_{h} \in$ [100 GeV, $130 \mathrm{GeV}]$

2.2. MVA-Based Method. We implemented the MVA method using the Root Toolkit for Multivariate Analysis (TMVA) [19]. After cut 1 , cut 2 , and cut 3 , we especially select several input variables to discriminate the signal and background events, thus resulting in better signal significance. Specifically, we define a set of totally 44 kinematic variables and choose the most effective ones for Boosted Decision Trees (BDT) training, which are the $b$-jet number $\left(N_{b j \mathrm{jet}}\right)$, the separation in the $\Phi-\eta$ plane between jets $\left(\Delta R^{B_{1} B_{2}}, \Delta R^{B_{1} J_{1}}\right)$, the difference in azimuthal angle between jets $\left(\Delta \Phi^{B_{1} B_{2}}, \Delta \Phi^{B_{1} J_{1}}\right)$, the transverse momentum of the jet $\left(p_{T}^{J_{1}}\right)$, and the difference in $|\eta|$ within Higgs jet system $\left(\Delta \eta^{h J_{1}}\right)$. It is worth noting that $e$-beam polarization is considered in both cut-based method and MVA-based method.

\section{Results}

In Figure $2((60) \mathrm{GeV})$ and Figure $3((120) \mathrm{GeV})$, we show the dependence of the cross section $\sigma$ on the top-Higgs FCNC couplings $\kappa_{t q h}$ at $E_{e}=60(120) \mathrm{GeV}$ with $p_{e}= \pm 0.6$ electron beam polarization and combination of a $7(50) \mathrm{GeV}$ proton beam for three different cases: (I) $\kappa_{t q h}=\kappa_{t u h}, \kappa_{t c h}=0$, (II) $\kappa_{t q h}=\kappa_{t c h}, \kappa_{t u h}=0$, and (III) $\kappa_{t q h}=\kappa_{t u h}=\kappa_{t c h}$. Obviously, the cross section of $\kappa_{t q h}=0.1$ can be 100 times larger than that of $\kappa_{t q h}=0.01$, and the cross section of $50 \mathrm{TeV}$ can be 9.1 (6.6) times larger than that of $7 \mathrm{TeV}$ with a 60 (120) GeV electron beam. We also find that the cross sections between polarized and unpolarized electron beam cases are related as $\sigma_{e_{r}^{-}}=\sigma_{e_{0}^{-}}$. $\left(1-p_{e_{r}^{-}}\right)$and $\sigma_{e_{l}^{-}}+\sigma_{e_{r}^{-}}=2 \sigma_{e_{0}^{-}}$, independent of being case I, case II, or caseIII. Here $\sigma_{e_{r}^{-}}, \sigma_{e_{l}^{-}}$, and $\sigma_{e_{0}^{-}}$represent the right, left, and without electron beam polarization, respectively.

The cross section of the signal and backgrounds (in units of $\mathrm{fb}$ ) are summarized in Table 2 (cut-based method) and Table 3 (MVA-based method). From these tables, we calculate the signal significance $S / \sqrt{S+B}$ as 4.191 (15.341) and 6.652 (19.236) for 7 and $50 \mathrm{TeV}$ by cut-based method and 4.921 (16.934) and 7.874 (20.785) by MVA-based method after imposing all the relevant event selections (only the first three selections in MVA-based method), respectively. Obviously, compared to the cut-based method, the MVA-based method can get better signal significance. As expected, with the $p_{2}=-0.6 e$-beam polarization, the results are improved as 5.302 (19.404) and 8.414 (24.335) for cut-based method and 6.224 (21.420) and 9.960 (26.291) for MVA-based method. In addition to effective cuts, enhancing the $b$-tagging efficiency together with reducing the jet misidentification rates is one of the other ways to improve the signal significance. It is confirmed that the signal significance can be increased from $4.191,6.652,15.341$, and 19.238 to $8.366,13.840,33.750$, and 44.154 with $\epsilon_{b}=80 \%, \epsilon_{c}=1 \%$, and $\epsilon_{\text {light }}=0.1 \%$ with the same value of the input parameters and kinematic cuts. 

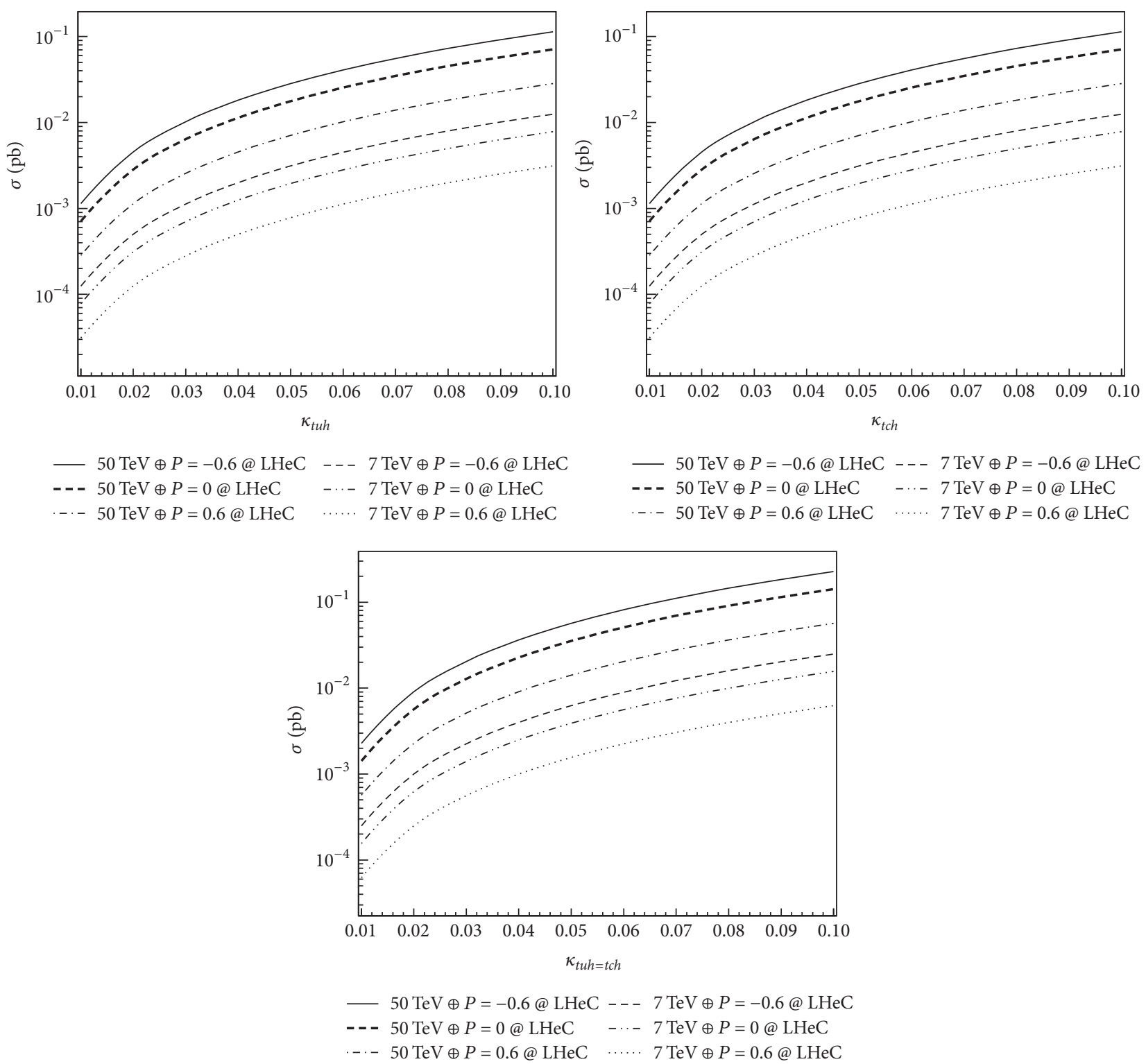

Figure 2: The cross sections $\sigma_{\text {tqh }}$ on the top-Higgs FCNC couplings $\kappa_{t q h}$ at the 7 (50) TeV and $60 \mathrm{GeV}$ LHeC with $e$-beam polarization $p_{e}=0, \pm 0.6$.

In order to estimate the sensitivity to the anomalous tqH couplings, we used chi-square $\left(\chi^{2}\right)$ function $[20,21]$ :

$$
\chi^{2}=\left(\frac{\sigma_{\text {tot }}-\sigma_{B}}{\sigma_{B} \delta}\right)^{2}
$$

where $\sigma_{\text {tot }}$ is the total cross section and $\delta$ is the statistical error. In Figure 4 (cut-based analysis) and Figure 5 (MVA-based analysis) [19], we plot the contours of $1 \sigma$ limits to $\kappa_{t q H}$ at 7 (50) GeV LHeC and 60 (120) GeV electron beam with different polarization. The red, blue, and black curves represent the $0.6,-0.6$, and without electron beam polarization. From these figures, we can see that the branching ratio $\mathrm{Br}(t \rightarrow u h)$ can be probed to $0.113(0.093 \%), 0.071(0.057 \%), 0.030(0.022 \%)$, and $0.024(0.019 \%)$ with the cut-based (MVA-based) analysis at $\left(E_{p}, E_{e}\right)=(7 \mathrm{TeV}, 60 \mathrm{GeV}),\left(E_{p}, E_{e}\right)=(7 \mathrm{TeV}, 120 \mathrm{GeV})$, $\left(E_{p}, E_{e}\right)=(50 \mathrm{TeV}, 60 \mathrm{GeV})$, and $\left(E_{p}, E_{e}\right)=(50 \mathrm{TeV}, 120 \mathrm{GeV})$ beam energy. As expected, the MVA-based method has a great advantage and also the $50 \mathrm{TeV}$ high energy can get better results compared to the $7 \mathrm{TeV}$ ones. Furthermore, it is clear that the limits can be probed down to 0.090 (0.073\%), 0.056 $(0.045 \%), 0.024(0.018 \%)$, and $0.019(0.015 \%)$ with the $e$-beam polarization of $p_{2}=-0.6$.

Finally, we give precise integrated luminosity $(\mathscr{L})$ corresponding to the critical limits obtained by the experimental results (Table 4) and other phenomenological studies 

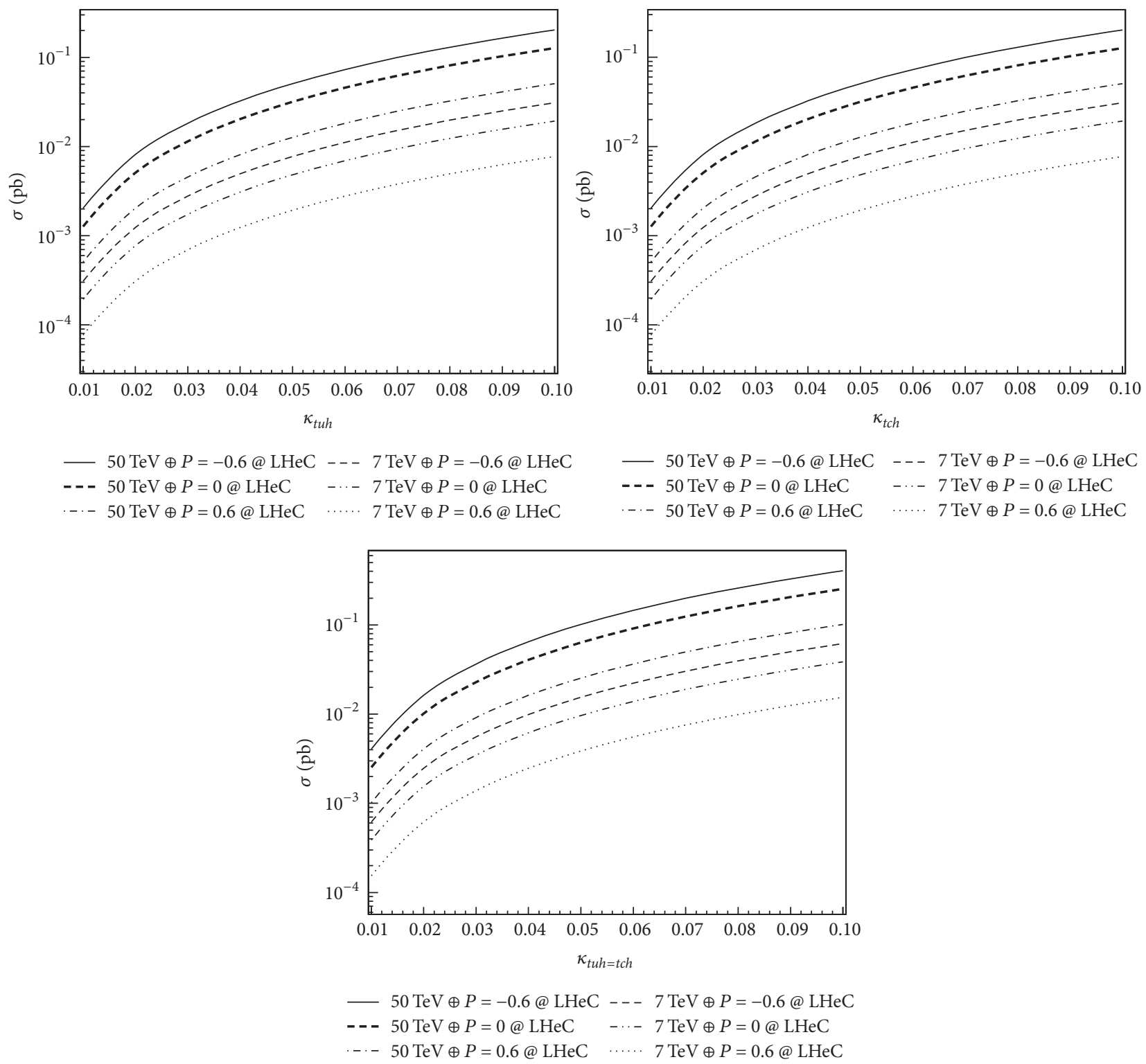

Figure 3: The same as Figure 2 but for $E_{e}=120 \mathrm{GeV}$.

(Table 5). With the $e$-beam polarization $p_{2}=-0.6, \mathscr{L}$ needed to get the upper bounds on $\operatorname{Br}(t \rightarrow q h)$ is reduced significantly. A detailed comparison between the LHeC collider(s) and the LHC or linear colliders is given.

\section{Conclusion}

In this paper, we investigated the anomalous FCNC Yukawa interactions between the top quark, the Higgs boson, and either an up or charm quark with a channel $e^{-} p \rightarrow$ $v_{e} \bar{t} \rightarrow v_{e} h \bar{q}(h \rightarrow b \bar{b})$ at the LHeC. The signal significance $S / \sqrt{S+B}$ can be obtained as 4.191 (4.921), 6.652 (7.874), 15.341 (16.934), and 19.238 (20.785) with the cut-based (MVA-based) method at $\left(E_{p}, E_{e}\right)=(7 \mathrm{TeV}, 60 \mathrm{GeV}),\left(E_{p}, E_{e}\right)=(7 \mathrm{TeV}$,
$120 \mathrm{GeV}),\left(E_{p}, E_{e}\right)=(50 \mathrm{TeV}, 60 \mathrm{GeV})$, and $\left(E_{p}, E_{e}\right)=(50 \mathrm{TeV}$, $120 \mathrm{GeV})$. Similarly, our results show that the branching ratio $\mathrm{Br}(t \rightarrow u h)$ can be probed to $0.113(0.093 \%), 0.071$ $(0.057 \%), 0.030(0.022 \%)$, and $0.024(0.019 \%)$, and with the $e$-beam polarization $p_{2}=-0.6$, the expected limits can be greatly reduced. Finally, a detailed comparison between our study and the critical limits obtained by the experiments and other phenomenological studies is shown. We thus give an overview of the search potential on the anomalous top-Higgs couplings with polarized electron beam at the LHeC.

\section{Conflicts of Interest}

The authors declare that they have no Conflicts of interest. 

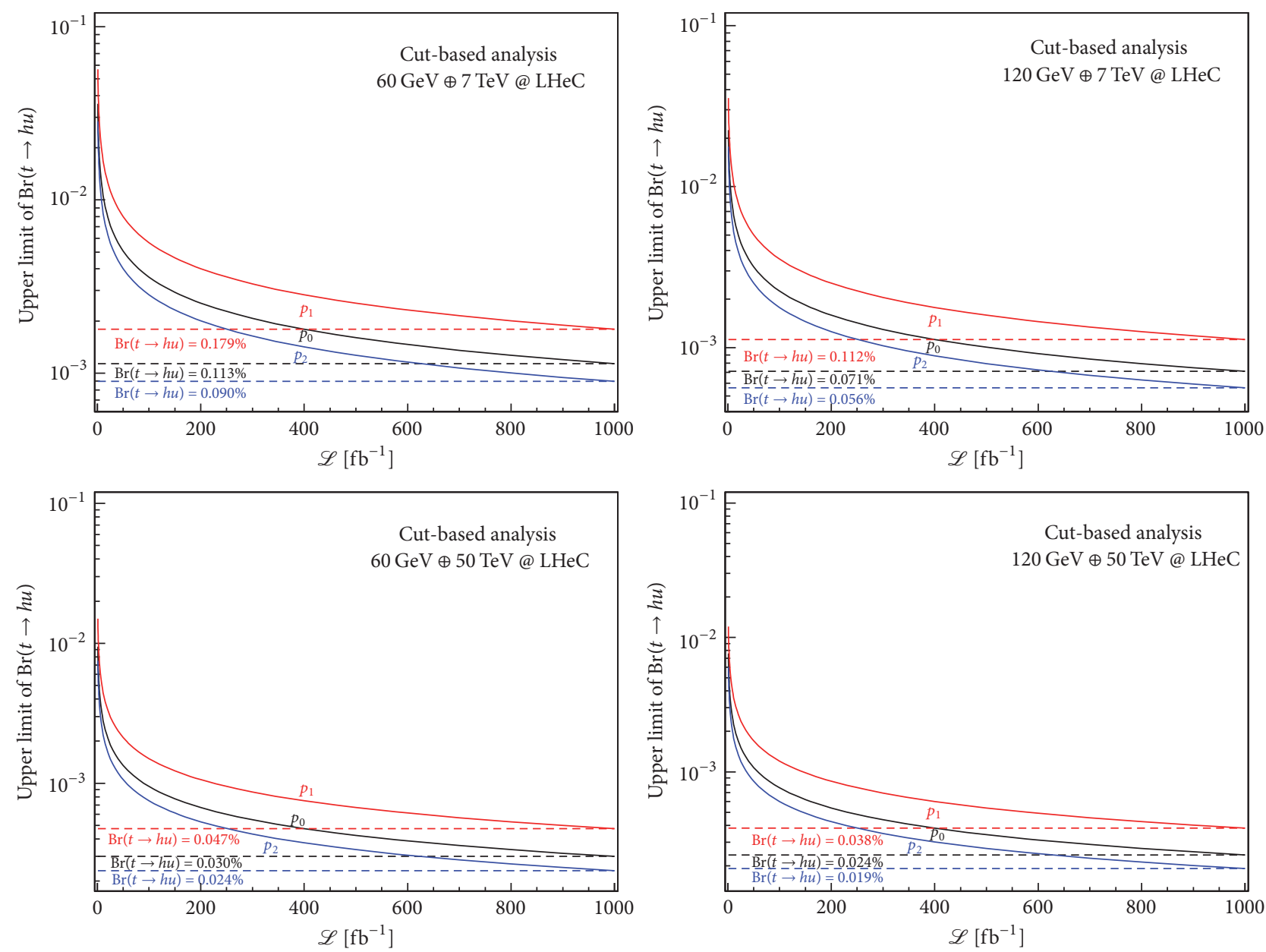

Figure 4: The upper limit from cut-based method at $1 \sigma$ level at 7 (50) GeV LHeC with 60 (120) GeV electron beam. The red, blue, and black curves represent the $0.6,-0.6$, and without electron beam polarization.

TABLE 2: Expected cross sections after all the selections for signal and backgrounds at the LHeC with integrated luminosity of $1000 \mathrm{fb}^{-1}, b$ tagging efficiency $\epsilon_{b}=60 \%$, jet misidentification rates $\epsilon_{c}=10 \%$, and $\epsilon_{\text {light }}=1 \%$ by cut-based method. In particular, we select $e$-beam polarization as $p_{0}=0, p_{1}=0.6$, and $p_{2}=-0.6$.

\begin{tabular}{|c|c|c|c|}
\hline & $S$ & $B$ & SS \\
\hline \multicolumn{4}{|c|}{$60 \mathrm{GeV} \oplus 7 \mathrm{TeV} @ \mathrm{LHeC}$} \\
\hline$p_{0}$ & 0.14 & 0.93 & 4.191 \\
\hline$p_{1}$ & 0.05 & 0.37 & 2.651 \\
\hline$p_{2}$ & 0.22 & 1.49 & 5.302 \\
\hline \multicolumn{4}{|c|}{$120 \mathrm{GeV} \oplus 7 \mathrm{TeV} @ \mathrm{LHeC}$} \\
\hline$p_{0}$ & 0.32 & 1.98 & 6.652 \\
\hline$p_{1}$ & 0.13 & 0.79 & 4.207 \\
\hline$p_{2}$ & 0.51 & 3.16 & 8.414 \\
\hline \multicolumn{4}{|c|}{$60 \mathrm{GeV} \oplus 50 \mathrm{TeV} @ \mathrm{LHeC}$} \\
\hline$p_{0}$ & 1.29 & 5.80 & 15.341 \\
\hline$p_{1}$ & 0.52 & 2.32 & 9.702 \\
\hline$p_{2}$ & 2.07 & 9.28 & 19.404 \\
\hline \multicolumn{4}{|c|}{$120 \mathrm{GeV} \oplus 50 \mathrm{TeV} @ \mathrm{LHeC}$} \\
\hline$p_{0}$ & 2.14 & 10.26 & 19.238 \\
\hline$p_{1}$ & 0.86 & 4.10 & 12.167 \\
\hline$p_{2}$ & 3.43 & 16.42 & 24.335 \\
\hline
\end{tabular}



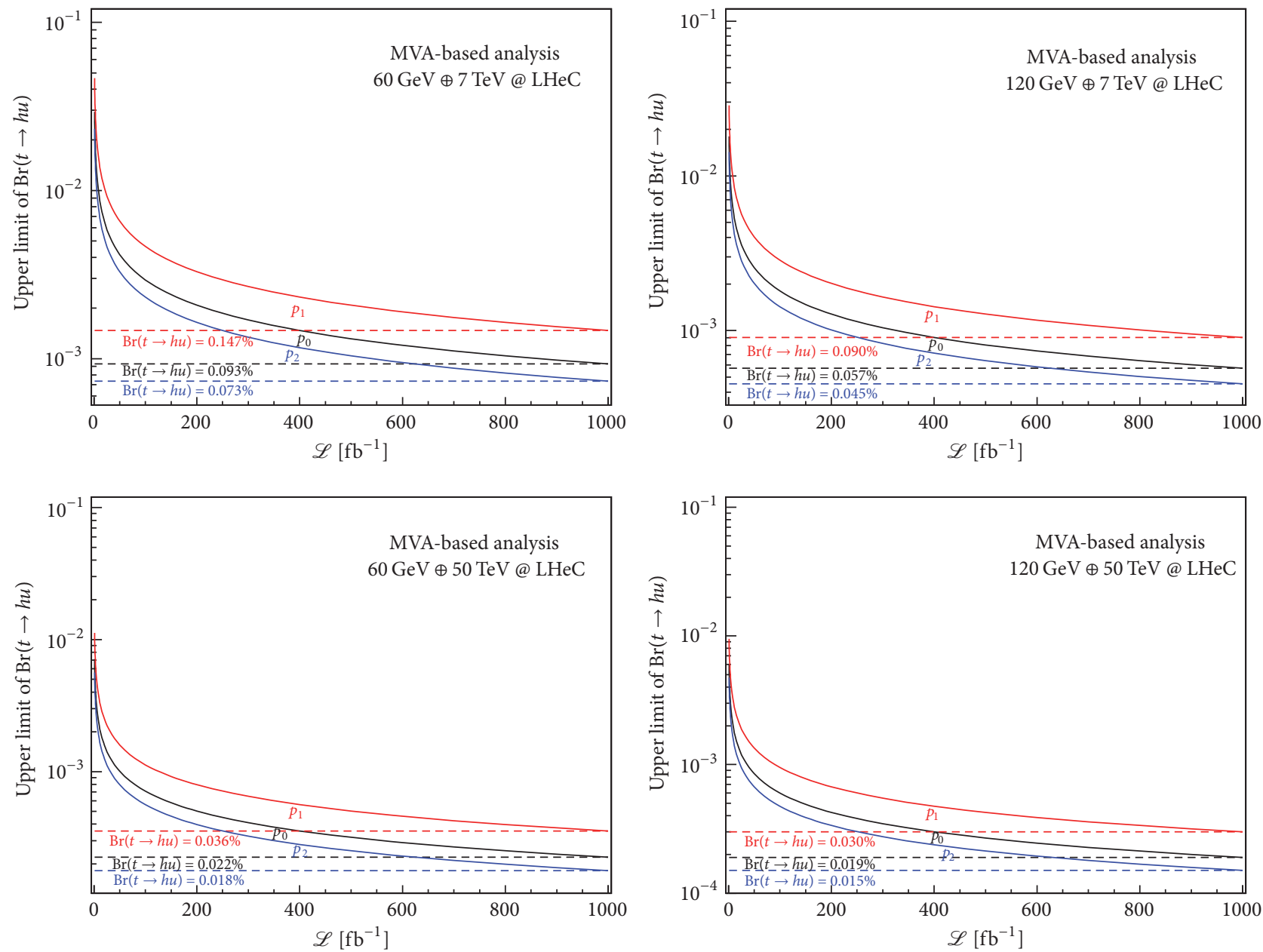

FIgURE 5: The same as Figure 4 but for MVA.

TABLE 3: The same as Table 2 but for MVA-based method. We select $e$-beam polarization as $p_{0}=0, p_{1}=0.6$, and $p_{2}=-0.6$.

\begin{tabular}{|c|c|c|c|}
\hline & $S$ & $B$ & SS \\
\hline \multicolumn{4}{|c|}{$60 \mathrm{GeV} \oplus 7 \mathrm{TeV} @ \mathrm{LHeC}$} \\
\hline$p_{0}$ & 0.125 & 0.520 & 4.921 \\
\hline$p_{1}$ & 0.050 & 0.208 & 3.112 \\
\hline$p_{2}$ & 0.200 & 0.833 & 6.224 \\
\hline \multicolumn{4}{|c|}{$120 \mathrm{GeV} \oplus 7 \mathrm{TeV} @ \mathrm{LHeC}$} \\
\hline$p_{0}$ & 0.281 & 0.992 & 7.874 \\
\hline$p_{1}$ & 0.112 & 0.397 & 4.980 \\
\hline$p_{2}$ & 0.450 & 1.588 & 9.960 \\
\hline \multicolumn{4}{|c|}{$60 \mathrm{GeV} \oplus 50 \mathrm{TeV} @ \mathrm{LHeC}$} \\
\hline$p_{0}$ & 0.652 & 0.830 & 16.934 \\
\hline$p_{1}$ & 0.261 & 0.332 & 10.710 \\
\hline$p_{2}$ & 1.043 & 1.328 & 21.420 \\
\hline \multicolumn{4}{|c|}{$120 \mathrm{GeV} \oplus 50 \mathrm{TeV} @ \mathrm{LHeC}$} \\
\hline$p_{0}$ & 1.082 & 1.629 & 20.785 \\
\hline$p_{1}$ & 0.433 & 0.652 & 13.145 \\
\hline$p_{2}$ & 1.732 & 2.606 & 26.291 \\
\hline
\end{tabular}


TABLE 4: The integrated luminosity $(\mathscr{L})$ needed to get the upper bounds on $\operatorname{Br}(t \rightarrow q h)$ at 95\% CL obtained from the experiments. Both the cut- (MVA-) based results and $1 \sigma(2 \sigma)$ limits with $e$-beam polarization are presented.

\begin{tabular}{|c|c|c|c|c|c|c|c|}
\hline \multirow[t]{2}{*}{ Channels and limits } & \multirow[t]{2}{*}{ Method } & \multicolumn{3}{|c|}{$\mathscr{L}\left[\mathrm{fb}^{-1}\right]_{1 \sigma}$} & \multicolumn{3}{|c|}{$\mathscr{L}\left[\mathrm{fb}^{-1}\right]_{2 \sigma}$} \\
\hline & & $p_{0}$ & $p_{1}$ & $p_{2}$ & $p_{0}$ & $p_{1}$ & $p_{2}$ \\
\hline $\begin{array}{l}t \bar{t} \rightarrow W b q h \rightarrow \ell v b \gamma \gamma q \\
\text { ATLAS, } 4.7(20.3) \mathrm{fb}^{-1} @ 7(8) \mathrm{TeV} \\
\operatorname{Br}(t \rightarrow q h)<0.79 \%[5,6]\end{array}$ & $\begin{array}{c}\text { Cut } \\
\text { MVA }\end{array}$ & $\begin{array}{l}0.93 \\
0.58\end{array}$ & $\begin{array}{l}2.32 \\
1.44\end{array}$ & $\begin{array}{l}0.58 \\
0.36\end{array}$ & $\begin{array}{l}3.60 \\
2.24\end{array}$ & $\begin{array}{l}9.00 \\
5.60\end{array}$ & $\begin{array}{l}2.25 \\
1.40\end{array}$ \\
\hline 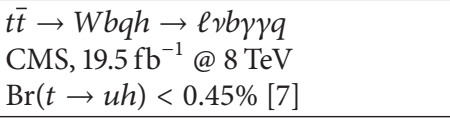 & $\begin{array}{l}\text { Cut } \\
\text { MVA }\end{array}$ & $\begin{array}{l}2.86 \\
1.78\end{array}$ & $\begin{array}{l}7.15 \\
4.45\end{array}$ & $\begin{array}{l}1.79 \\
1.11\end{array}$ & $\begin{array}{l}11.10 \\
6.91\end{array}$ & $\begin{array}{l}27.76 \\
17.27\end{array}$ & $\begin{array}{l}6.94 \\
4.32\end{array}$ \\
\hline $\begin{array}{l}D^{0}-\overline{D^{0}} \text { mixing data } \\
\operatorname{Br}(t \rightarrow q h)<0.5 \%[8]\end{array}$ & $\begin{array}{l}\text { Cut } \\
\text { MVA }\end{array}$ & $\begin{array}{l}2.32 \\
1.44\end{array}$ & $\begin{array}{l}5.79 \\
3.60\end{array}$ & $\begin{array}{l}1.45 \\
0.90\end{array}$ & $\begin{array}{l}8.99 \\
5.60\end{array}$ & $\begin{array}{l}22.48 \\
13.99\end{array}$ & $\begin{array}{l}5.62 \\
3.50\end{array}$ \\
\hline $\begin{array}{l}Z \rightarrow c \bar{c} \text { and EW observables } \\
\operatorname{Br}(t \rightarrow q h)<0.21 \%[9]\end{array}$ & $\begin{array}{l}\text { Cut } \\
\text { MVA }\end{array}$ & $\begin{array}{c}13.13 \\
8.17\end{array}$ & $\begin{array}{l}32.83 \\
20.43\end{array}$ & $\begin{array}{l}8.21 \\
5.11\end{array}$ & $\begin{array}{l}51.01 \\
31.74\end{array}$ & $\begin{array}{l}127.53 \\
79.35\end{array}$ & $\begin{array}{l}31.88 \\
19.84\end{array}$ \\
\hline
\end{tabular}

TABLE 5: The same as Table 4 but for some other phenomenological studies.

\begin{tabular}{|c|c|c|c|c|c|c|c|}
\hline \multirow[t]{2}{*}{ Channels and limits } & \multirow{2}{*}{ Method } & \multicolumn{3}{|c|}{$\mathscr{L}\left[\mathrm{fb}^{-1}\right]_{1 \sigma}$} & \multicolumn{3}{|c|}{$\mathscr{L}\left[\mathrm{fb}^{-1}\right]_{2 \sigma}$} \\
\hline & & $p_{0}$ & $p_{1}$ & $p_{2}$ & $p_{0}$ & $p_{1}$ & $p_{2}$ \\
\hline $\begin{array}{l}\mathrm{Wt} \rightarrow W h q \rightarrow \ell v b \gamma \gamma q \\
\mathrm{LHC}, 3000 \mathrm{fb}^{-1} @ 14 \mathrm{TeV} \\
3 \sigma, \mathrm{Br}(t \rightarrow q h)<0.24 \%[10]\end{array}$ & $\begin{array}{c}\text { Cut } \\
\text { MVA }\end{array}$ & $\begin{array}{c}10.05 \\
6.26\end{array}$ & $\begin{array}{l}25.14 \\
15.64\end{array}$ & $\begin{array}{l}6.28 \\
3.91\end{array}$ & $\begin{array}{l}39.05 \\
24.30\end{array}$ & $\begin{array}{l}97.63 \\
60.75\end{array}$ & $\begin{array}{l}24.41 \\
15.19\end{array}$ \\
\hline $\begin{array}{l}t \bar{t} \rightarrow W b q h \rightarrow \ell v b \gamma \gamma q \\
\mathrm{LHC}, 3000 \mathrm{fb}^{-1} @ 14 \mathrm{TeV} \\
\operatorname{Br}(t \rightarrow u h)<0.23 \%[11] \\
\end{array}$ & $\begin{array}{c}\text { Cut } \\
\text { MVA }\end{array}$ & $\begin{array}{c}10.95 \\
6.81\end{array}$ & $\begin{array}{l}27.37 \\
17.03\end{array}$ & $\begin{array}{l}6.84 \\
4.26\end{array}$ & $\begin{array}{l}42.52 \\
26.46\end{array}$ & $\begin{array}{c}106.31 \\
66.15\end{array}$ & $\begin{array}{l}26.58 \\
16.54\end{array}$ \\
\hline $\begin{array}{l}t \bar{t} \rightarrow t q h \rightarrow \ell \nu b b \bar{b} q \\
\mathrm{ILC}, 3000 \mathrm{fb}^{-1} @ 500 \mathrm{GeV} \\
\operatorname{Br}(t \rightarrow q h)<0.112 \%[12]\end{array}$ & $\begin{array}{c}\text { Cut } \\
\text { MVA }\end{array}$ & $\begin{array}{l}46.20 \\
28.75\end{array}$ & $\begin{array}{c}115.50 \\
71.86\end{array}$ & $\begin{array}{l}28.87 \\
17.97\end{array}$ & $\begin{array}{c}179.44 \\
111.65\end{array}$ & $\begin{array}{c}448.60 \\
279.13\end{array}$ & $\begin{array}{l}112.15 \\
69.78\end{array}$ \\
\hline $\begin{array}{l}\text { th } \rightarrow \ell \nu b \tau^{+} \tau^{-} \\
\mathrm{LHC}, 100 \mathrm{fb}^{-1} @ 13 \mathrm{TeV} \\
\operatorname{Br}(t \rightarrow u h)<0.15 \%[13]\end{array}$ & $\begin{array}{c}\text { Cut } \\
\text { MVA }\end{array}$ & $\begin{array}{l}25.75 \\
16.02\end{array}$ & $\begin{array}{l}64.37 \\
40.05\end{array}$ & $\begin{array}{l}16.09 \\
10.01\end{array}$ & $\begin{array}{c}100.01 \\
62.23\end{array}$ & $\begin{array}{l}250.03 \\
155.58\end{array}$ & $\begin{array}{l}62.51 \\
38.89\end{array}$ \\
\hline $\begin{array}{l}\text { th } \rightarrow \ell \nu b \ell^{+} \ell^{-} X \\
\mathrm{LHC}, 100 \mathrm{fb}^{-1} @ 13 \mathrm{TeV} \\
\operatorname{Br}(t \rightarrow u h)<0.22 \%[13] \\
\end{array}$ & $\begin{array}{c}\text { Cut } \\
\text { MVA }\end{array}$ & $\begin{array}{c}11.97 \\
7.45\end{array}$ & $\begin{array}{l}29.92 \\
18.61\end{array}$ & $\begin{array}{l}7.48 \\
4.65\end{array}$ & $\begin{array}{l}46.48 \\
28.92\end{array}$ & $\begin{array}{l}116.20 \\
72.30\end{array}$ & $\begin{array}{l}29.05 \\
18.08\end{array}$ \\
\hline $\begin{array}{l}\text { th } \rightarrow j j b b \bar{b} \\
\mathrm{LHC}, 100 \mathrm{fb}^{-1} @ 13 \mathrm{TeV} \\
\operatorname{Br}(t \rightarrow u h)<0.36 \%[13]\end{array}$ & $\begin{array}{c}\text { Cut } \\
\text { MVA }\end{array}$ & $\begin{array}{l}4.47 \\
2.78\end{array}$ & $\begin{array}{l}11.17 \\
6.95\end{array}$ & $\begin{array}{l}2.79 \\
1.74\end{array}$ & $\begin{array}{c}17.35 \\
10.80\end{array}$ & $\begin{array}{l}43.38 \\
26.99\end{array}$ & $\begin{array}{c}10.84 \\
6.75\end{array}$ \\
\hline
\end{tabular}

\section{Acknowledgments}

This project is supported by the National Natural Science Foundation of China (Grant no. 11675033) and by the Fundamental Research Funds for the Central Universities (Grant no. DUT15LK22).

\section{References}

[1] O. Brüning and M. Klein, "The large hadron electron collider," Modern Physics Letters A, vol. 28, no. 16, Article ID 1330011, 2013.

[2] M. Klein, "Development of the FCC-he study," in Proceedings of the FCC Physics, Detector and Accelerator Workshop, Istanbul, Turkey, March 2016.

[3] C. S. Li, R. J. Oakes, and T. C. Yuan, "QCD corrections to $t \rightarrow$ $W^{+} \rightarrow$ b," Physical Review D, vol. 43, no. 11, article 3759, 1991.

[4] W.-S. Hou, "Tree level $t \rightarrow c h$ or $h \rightarrow t^{-} c$ decays," Physics Letters $B$, vol. 296, pp. 179-184, 1992.

[5] ATLAS Collaboration, "Search for top quark decays $t \rightarrow q H$ with $H \rightarrow \gamma \gamma$ using the ATLAS detector," JHEP, vol. 6, 2014.
[6] ATLAS Collaboration, "Search for flavour changing neutral currents in top quark decays $t \rightarrow c H$, with $H \rightarrow \gamma \gamma$, and limit on the tcH coupling with the ATLAS detector at the LHC,' Tech. Rep. ATLAS-CONF-2013-081, 2013.

[7] V. Khachatryan, A. M. Sirunyan, A. Tumasyan et al., "Searches for heavy Higgs bosons in two-Higgs-doublet models and for $t \rightarrow c h$ decay using multilepton and diphoton final states in $p p$ collisions at 8 TeV," Physical Review D, vol. 90, no. 11, Article ID 112013, 23 pages, 2014.

[8] J. I. Aranda, A. Cordero-Cid, F. Ramirez-Zavaleta, J. J. Toscano, and E. S. Tututi, "Higgs mediated flavor violating top quark decays $t \rightarrow u_{i} H, u_{i} \gamma, u_{i} \gamma \gamma$ and the process $\gamma \gamma \rightarrow t c$ in effective theories," Physical Review D, vol. 81, Article ID 077701, 2010.

[9] F. Larios, R. Martinez, and M. A. Perez, "Constraints on topquark flavor changing neutral couplings from electroweak precision measurements," Physical Review D, vol. 72, no. 5, Article ID 057504, 4 pages, 2005.

[10] Y.-B. Liu and Z.-J. Xiao, "Searches for top-Higgs FCNC couplings via the Whj signal with $h \rightarrow \gamma \gamma$ at the LHC," Physical Review D, vol. 94, no. 5, Article ID 054018, 2016. 
[11] L. Wu, "Enhancing thj production from Top-Higgs FCNC couplings," Journal of High Energy Physics, vol. 2015, no. 2, article 061, 2015.

[12] H. Hesari, H. Khanpour, and M. M. Najafabadi, "Direct and indirect searches for top-Higgs FCNC couplings," Physical Review D, vol. 92, no. 11, Article ID 113012, 2015.

[13] A. Greljo, J. F. Kamenik, and J. Kopp, "Disentangling flavor violation in the top-Higgs sector at the LHC," JHEP, vol. 1407, article 46, 2014.

[14] A. Alloul, N. D. Christensen, C. Degrande, C. Duhr, and B. Fuks, "FeynRules 2.0-a complete toolbox for tree-level phenomenology," Computer Physics Communications, vol. 185, no. 8, pp. 2250-2300, 2014.

[15] J. Alwall, R. Frederix, S. Frixione et al., “The automated computation of tree-level and next-to-leading order differential cross sections, and their matching to parton shower simulations," Journal of High Energy Physics, vol. 2014, no. 7, article no. 079, 2014.

[16] T. Sjöstrand, S. Mrenna, and P. Skands, "PYTHIA 6.4 physics and manual," Journal of High Energy Physics, vol. 2006, no. 5, article no. 026, 2006.

[17] J. Pumplin, D. R. Stump, J. Huston, H.-L. Lai, P. Nadolsky, and W.-K. Tung, "New generation of parton distributions with uncertainties from global QCD analysis," Journal of High Energy Physics, vol. 2002, no. 7, article no. 012, 2002.

[18] D. Stump, J. Huston, J. Pumplin et al., "Inclusive jet production, parton distributions, and the search for new physics," Journal of High Energy Physics, vol. 2003, no. 10, article 046, 2003.

[19] A. Hoecker, P. Speckmayer, J. Stelzer et al., "TMVA-toolkit for multivariate data analysis," PoS ACAT 040, CERN-OPEN2007-007, 2007.

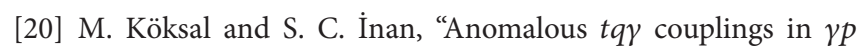
collision at the LHC," Advances in High Energy Physics, vol. 2014, Article ID 935840, 11 pages, 2014.

[21] H. Sun, "Probe anomalous tq $\gamma$ couplings through single top photoproduction at the LHC", Nuclear Physics B, vol. 886, pp. 691-711, 2014. 

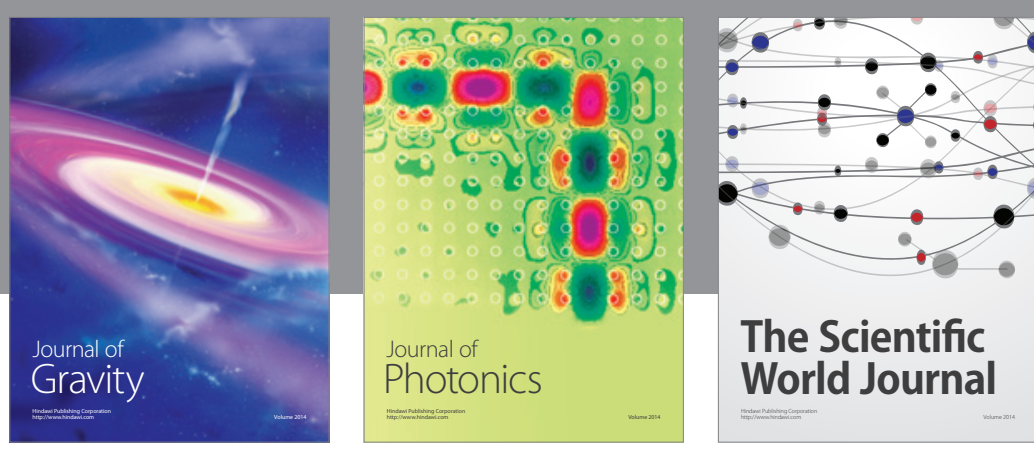

The Scientific World Journal
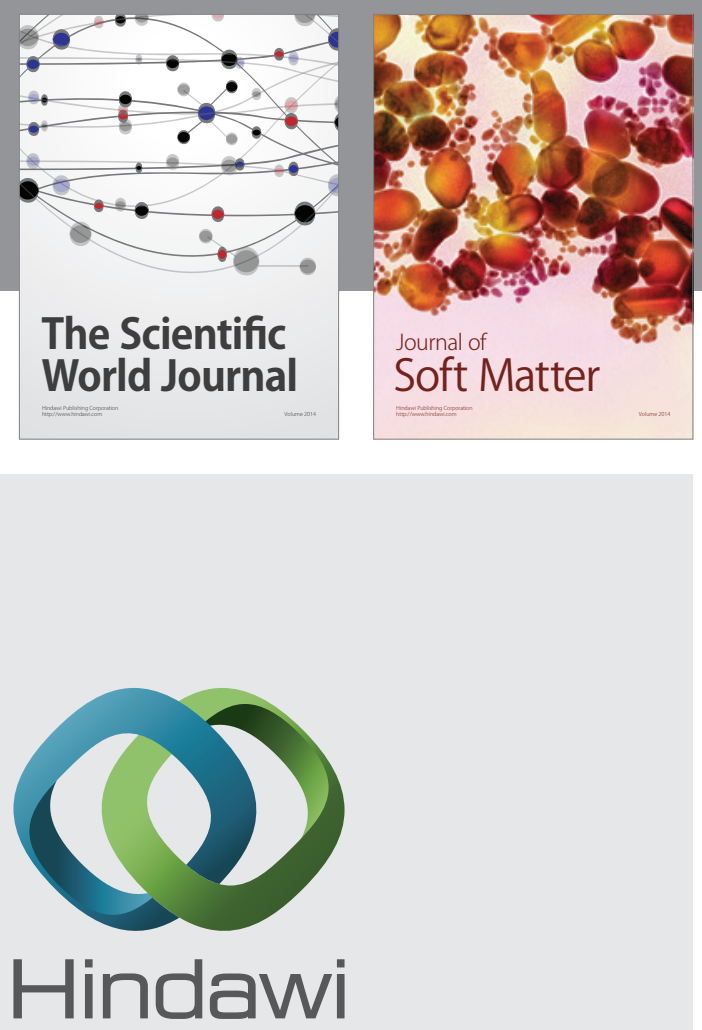

Submit your manuscripts at

https://www.hindawi.com
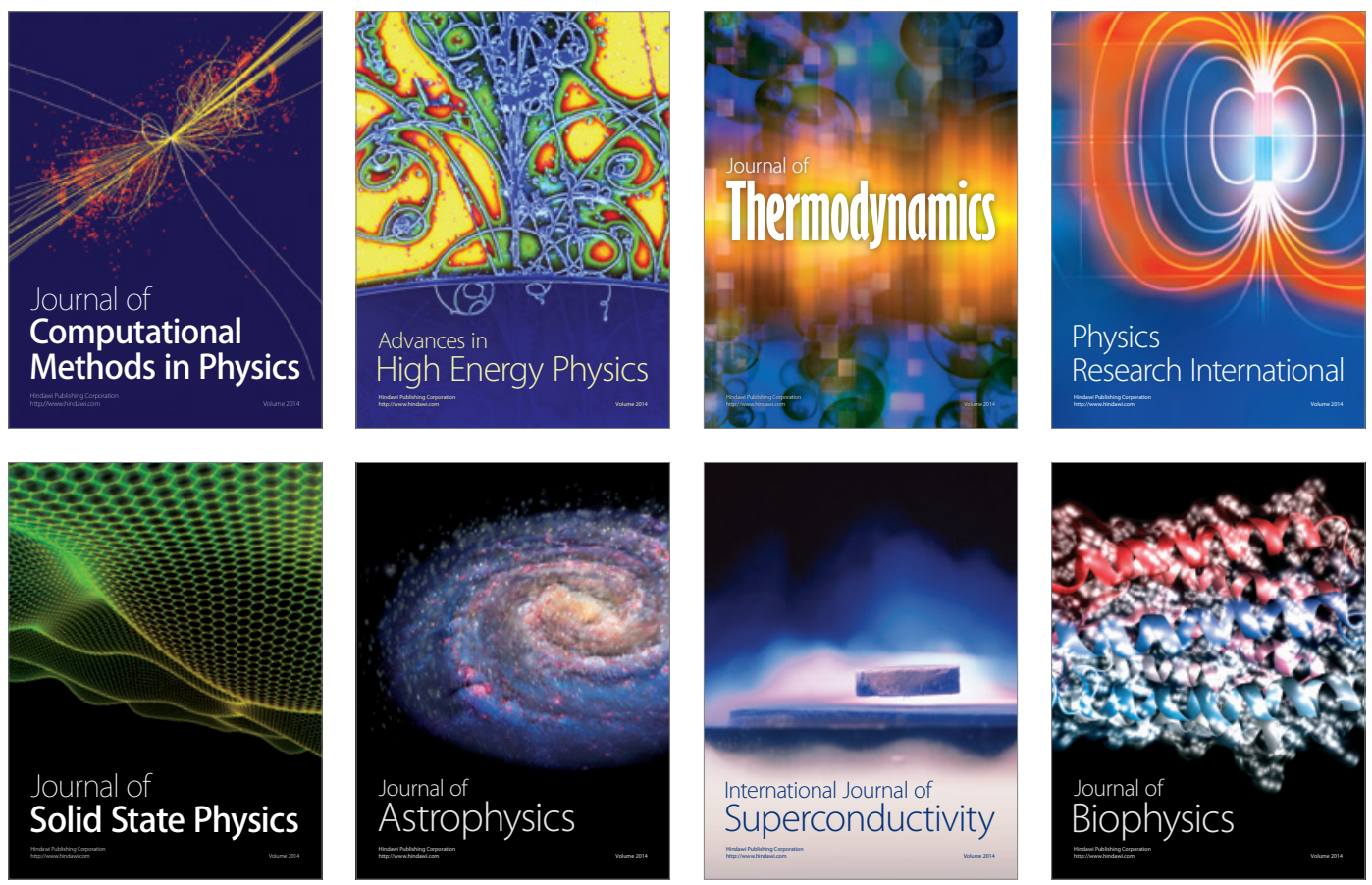
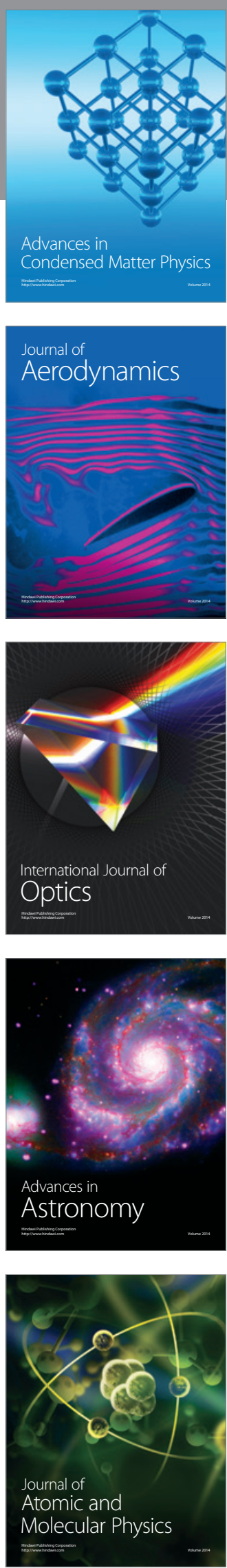